\title{
Role of ACE 2 and Vitamin D: The Two Players in Global Fight against COVID-19 Pandemic
}

\author{
Tapan Behl ${ }^{1}$ Sadia Shah ${ }^{2}$ Ishnoor Kaur ${ }^{1} \quad$ Sushma Yadav ${ }^{3}$ \\ Naveet Wig ${ }^{6}$ K. K. Sharma ${ }^{7}$ Harlokesh Narayan Yadav ${ }^{2}$ \\ 1Department of Pharmacology, Chitkara University, Punjab, India \\ 2Department of Pharmacology, All India Institute of Medical \\ Sciences, New Delhi, India \\ ${ }^{3}$ Department of Obstetrics and Gynaecology, Shaheed Hasan Khan \\ Mewati Government Medical College, Haryana, India \\ ${ }^{4}$ Department of Nephrology, All India Institute of Medical Sciences, \\ New Delhi, India \\ ${ }^{5}$ Department of Cardiology, All India Institute of Medical Sciences, \\ New Delhi, India \\ ${ }^{6}$ Department of Medicine, All India Institute of Medical Sciences, \\ New Delhi, India \\ ${ }^{7}$ National Academy of Medical Sciences (India), New Delhi, India
}

Raj Kanwar ${ }^{4}$ Sandeep Seth ${ }^{5}$

Address for correspondence Harlokesh Narayan Yadav, PhD, Department of Pharmacology, All India Institute of Medical Sciences, New Delhi 110029, India (e-mail: hnyadav@gmail.com).

\begin{abstract}
Keywords

- ACE2

- molecular target

- angiotensin II

- ACE inhibitors

- ARBs

- vitamin D

- calcitriol

The global pandemic of coronavirus disease 2019 (COVID-19) has spread across the borders, gaining attention from both health care professional and researchers to understand the mode of entry and actions induced by severe acute respiratory syndrome coronavirus 2 (SARS-CoV-2), its causative agent in the human body. The role of angiotensin-converting enzyme-2 (ACE2) in facilitating the entry of the virus in the host cell by binding to it is similar to SARS-CoV-1, the causative agent for severe acute respiratory syndrome (SARS) which emerged in 2003. Besides the role of ACE2 as a molecular target for the virus, the review displays the potential benefits of ACE2 enzyme and various agents that modify its activity in curbing the effects of the deadly virus, thus unfolding a dual character of ACE2 in the current pandemic. As evident by the differences in the susceptibility toward viral infection in children and geriatric population, it must be noted that the older population has limited ACE2 levels and greater infection risk, whereas the situation is reversed in the case of the pediatric population, demonstrating the defensive character of ACE2 in the latter, despite acting as receptor target for SARS-CoV-2. Also, the upregulation of ACE2 levels by estrogen has indicated greater resistance to infection in females than in the male human population. ACE2 is a carboxypeptidase, which degrades angiotensin II and counteracts its actions to protect against cardiovascular risks associated with the virus. Another contribution of this enzyme is supported by the role of circulating soluble ACE2, which acts as a receptor to bind the virus but does not mediate its actions, therefore blocking its interaction to membrane-bound ACE2 receptors. The review also shares the enhanced risks of developing COVID-19 infection by using ACE inhibitors and ARBs. However, both these agents have been reported to upregulate
\end{abstract}

published online June 9, 2021
DOI https://doi.org/

$10.1055 / \mathrm{s}-0041-1729781$

ISSN 0379-038X
C2021. National Academy of Medical Sciences (India).

This is an open access article published by Thieme under the terms of the Creative Commons Attribution-NonDerivative-NonCommercial-License, permitting copying and reproduction so long as the original work is given appropriate credit. Contents may not be used for commercial purposes, or adapted, remixed, transformed or built upon. (https://creativecommons.org/licenses/by-nc-nd/4.0/).

Thieme Medical and Scientific Publishers Pvt. Ltd. A-12, 2nd Floor, Sector 2, Noida-201301 UP, India 
ACE2 levels; yet, adequate evidence regarding their role is quite inconsistent in human studies. Furthermore, the role of vitamin D has been highlighted in regulating the immune system of the body through renin-angiotensin-aldosterone system (RAAS) inhibition, by downregulating host cell receptor expression to prevent virus attachment. Besides, vitamin D also acts through several other mechanisms like upregulating antimicrobial peptides, fighting against the proinflammatory milieu created by the invading virus, and interfering with the viral replication cycle as well as calcitriol-mediated blockage of CREB protein. Hypovitaminosis D is attributed to elevated risks of acute respiratory distress syndrome (ARDS), lung damage, and cardiovascular disorders, further increasing the severity of COVID-19 infection.

\section{Introduction}

The novel coronavirus ( $\mathrm{nCoV}$ ) named severe acute respiratory syndrome coronavirus 2 (SARS-CoV-2) and the infectious coronavirus disease 2019 (COVID-19), caused by it, has created a global health care emergency on account of its rapid transmission worldwide. The COVID-19 infection began from Wuhan, the capital city of Hubei province in China, rapidly spreading to other parts of the world. ${ }^{1}$ Recent updates of the COVID-19 pandemic indicate the total number of infected individuals to be $159,614,066$ with $3,318,013$ deaths all over the world [https://www.worldometers.info/coronavirus/] and this number is increasing day by day. Countries like the United States, Brazil, Russia, Spain, United Kingdom, Italy, France, Germany, India, and so forth, are currently the most affected nations worldwide [https://www.worldometers. info/coronavirus/]. The researchers all over the world have been investigating to develop appropriate treatment for the pandemic to contain the mortality rate and damage caused by the COVID-19 outbreak. Till now, no effective clinical therapy has been established for the treatment. ${ }^{2}$ However, certain therapies to mitigate the extent and progression of the virus have been tried; these include hydroxychloroquine and chloroquine (antimalarials), remdesivir (antivirals), and mRNA-1273 (vaccines), which have been developed after accommodating detailed knowledge about the life cycle, genetic sequence, mode of action, and transmission dynamics. ${ }^{3}$ However, such information also indicates knowledge about which patients are most susceptible to the infections mediated by SARS-CoV-2. It has been suggested that patients on angiotensin receptors blockers (ARBs), or angiotensin-converting enzyme (ACE) inhibitors (ACE-Is) carry greater risks to develop COVID-19 infection, based on the knowledge that SARS-CoV-2 uses highly prevalent ACE2 transmembrane enzyme in the respiratory tract and alveolar epithelium for entry into the nasobronchial and pulmonary cells to cause the diseases and this protein is upregulated by the ARBs and ACE-Is. Renin-angiotensin-aldosterone system (RAAS) is an important peptide peptidase-operated coalition that subserves a critical role in various physiological and pathological processes in multiple organs involved in cardiovascular, mitotic, and immune functions. Besides several proteases/peptidases in the human body, two peptidase-Ang converting enzymes-ACE (in the present context can be called ACE1) and ACE2-are important proteases that form angiotensin II (Ang-II) and angiotensin (1-7) (Ang 1-7), which by acting through their receptors-AT1/ AT2 and Mas receptor, respectively-produce their effects. Therefore, the therapeutic approaches which counteract the mechanistic interaction of SARS-CoV-2 and ACE2, an important player of RAAS, are capable of inducing a protective response in the COVID-19 infection. ${ }^{3}$ Recently approved in Europe and the United States, angiotensin II (Ang-II) is a renal function and blood pressure (BP)-regulating hormone, which functions as a vasopressor agent in patients with or without shock. ${ }^{4}$ In the current scenario of COVID-19, the role of Ang-II must be significantly explored to facilitate the development of an effective treatment paradigm.

\section{Some Controversies which Need to Be Reconciled}

Patients with comorbidities are found to be more susceptible to critical COVID-19 infectious stages. However, healthy and young patients have also been affected by COVID-19 disease, posing a significant challenge for health care scientists and researchers. ${ }^{5}$ Within 10 days of the infection, the patients who are more likely to undergo acute respiratory distress syndrome (ARDS) and respiratory failure and are admitted to the ICU. ${ }^{6}$ Besides respiratory failure, septic shock might occur in certain patients, resulting in increased mortality rate. ${ }^{7}$ A report based on Chinese study in a cohort of hospitalized patients established $1.1 \%$ incidence of shock in infected patients, which rose to $6.4 \%$ in patients with severe comorbidities. On the other hand, talking about the geriatric population, the population of ACE2 receptors goes down in tissues of such people, but ironically substantial evidence is showing them to be highly vulnerable to severity of disease progression and subsequent mortality as compared with the other age groups. ${ }^{8}$ Also, resistance to COVID-19 spread and better tolerance is also observed in females, because $17 \beta$-estradiol (E2) increases the synthesis and availability and activity of ACE2 via agonistic action on the estrogen receptor $\alpha$ $(E R \alpha)$, as well as in children, due to high baseline levels of ACE2. ${ }^{9}$ Therefore, this review aims to highlight the possible relationship of ACE2 with COVID-19 disease along with special focus on the role of vitamin D in mitigating the effects of coronavirus. This immunomodulator possesses significant antiviral properties against several viral infections like influenza virus and coronaviruses. This hypothesis is based upon 
numerous studies depicting the relationship between vitamin D deficiency and severity of COVID-19 infection. ${ }^{10}$ The anti-inflammatory actions of vitamin $\mathrm{D}$ exert a protective influence against the cytokine storm, which contributes to the severity of the infection. ${ }^{10}$ Furthermore, besides the contribution of vitamin $\mathrm{D}$ in the regulation of adaptive and innate immune system, it also inhibits the renin-angiotensin system (RAS) and boosts the immune system of the patient, strengthening the defense system against COVID-19 infection. ${ }^{11}$ This review also provides a detailed cascade of the role of vitamin $\mathrm{D}$ as a therapeutic approach in the current pandemic, following an economically favorable profile as well as an accessible strategy to retard the progression and extent of infection of COVID-19.

\section{Role of ACE2 in Basic Physiology and Defense against COVID-19}

ACE2 was first discovered 20 years ago in 2000 and is homologous to ACE. ${ }^{12,13}$ ACE2 is a 17 -amino acid, C-terminal membrane anchor, and $\mathrm{N}$-terminal signal-cleaving peptide enzyme which has $42 \%$ amino acid identity with ACE and has evolved through gene duplication. Angiotensin II (Ang II) is an active peptide that is responsible for vasoconstriction, aldosterone secretion, and profibrosis and proinflammatory actions in the body (- Figs. 1, 2). ${ }^{12-14}$ Effects of this peptide are counteracted by angiotensin-(1-7), a peptide cleaved by ACE2, which exhibits opposite properties to that of Ang II, ${ }^{15}$ like vasodilatory, anti-inflammatory, and antifibrosis actions by binding to a G-protein-coupled receptor (GPCR), called Mas receptor (-Fig. 1). ${ }^{16}$ Besides ACE2, aminopeptidases also deactivate angiotensin II by converting it to angiotensin III, which is capable of increasing natriuresis and vasodilation via interaction with AT2 receptors with 30 times more affinity than AT1 receptors. ${ }^{17,18}$ Angiotensin I is converted to angiotensin-(1-9) under the influence of ACE2. The actions of angiotensin-(1-9) are not quite known and it is further transformed into angiotensin-(1-7) under the influence of ACE. ${ }^{14}$ The RAAS system comprises dual function pathways, one acts as an "activator" and another as "inhibitor," where the former comprises of Ang II/ACE/AT1 receptor/aldosterone pathway, and the latter comprises Ang (1-7)/ACE2/Mas receptor pathway. The inhibitor pathway exerts opposite actions, counteracting the effects of Ang II through its vasodilator with anti-inflammatory and antioxidant properties. ${ }^{12}$

The extracellular region of the cell constitutes a catalytic domain of ACE2, which can be cleaved and released into the blood by a disintegrin and metalloproteinase domain 17 (ADAM17). ${ }^{19}$ The actions of the inhibitor system of RAAS have been explored to little extent as compared with the activator system; however, certain in vitro studies demonstrated a positive impact on the cardiovascular system, when activated, and possibly involving elevation of cGMP levels. ${ }^{19,20}$ Moreover, ACE2 can heteroligomerize with the AT1 receptors via Mas receptors, resulting in inhibition of the actions of Ang II, thus playing a significant role in the regulation of Ang II in the body (-Fig. 1). ${ }^{20}$ Various organs of the body, like brain, kidney, digestive tract, adipose tissue, heart, testicles, and so forth, sufficiently express ACE2 in the cells. ${ }^{21}$ Besides, the epithelial cells in the alveoli of the lungs are the primary cells associated with ACE2 expression. ${ }^{22}$

Low levels of ACE 2 due to any secondary factor disrupts the equilibrium between ACE/ACE2 which renders the latter incompetent to cleave the Ang II to Ang (1-7), hence shunting its favorable effects in ameliorating the inflammatory environment created by Ang II which otherwise remains unchecked. Ang II, other than being a potent vasoconstrictor, plays a pivotal role in increasing oxidative stress as well as the release of various cytokines and chemokines, triggering a complex pulmonary hyperinflammatory response which is commonly referred to as "cytokine storm," leading to increased severity of symptoms in the coronavirus-positive patients and, thereby, an enhancement in the related morbidity and mortality ${ }^{23}$ in such individuals. ${ }^{24} \mathrm{~A}$ good amount of evidence is now available regarding the release of various proinflammatory cytokines such as IFN $\alpha$, IFN $\gamma$, IL-6, IL-12, IL-18, IL-1 $\beta$, IL-33, TNF $\alpha$, and TGF $\beta$ and chemokines including CXCL2, CXCL3, CXCL5, CCL8, CCL9, CCL10 which are found in the system of the severely SARS-coV-2-infected individuals, which triggers a consequential systemic inflammatory response resulting in organ failure and subsequent death. ${ }^{25}$ Certain groups of investigators have also suggested utilizing the circulating soluble ACE2 to capture the viruses in the plasma and prevent their interaction and binding to the membrane ACE2 receptors, therefore minimizing the infection spread. ${ }^{14}$ To facilitate this approach, genetically engineered recombinant soluble ACE2 has been investigated in vitro studies for its potential applications as a therapeutic approach to neutralize the infecting $\mathrm{nCoV}^{24}$ Furthermore, soluble ACE2 might favor the inhibitory pathway of RAAS over the activator pathway, thus promoting the treatment of inflammatory tissue lesions. ${ }^{26}$ The SARS-CoV-2 is shown to downregulate the expression of ACE2 by binding to it, resulting in accelerated lung damage. ${ }^{24}$ To protect ARDS during COVID-19 infection, the ACE2/angiotensin-(1-7) axis may, thus, be exploited to exert a protective response. ${ }^{19,20}$ Therefore, in recent years recombinant human ACE2 (rhACE2), like APN01 and GSK2586881, has attracted massive interest of the researchers. ${ }^{27,28}$ The rhACE2 has been found to abbreviate Ang II concentrations in the plasma and enhance the levels of Ang (1-7), resulting in prevention of myocardial fibrosis and hypertrophy as well as diastolic dysfunction, induced by Ang II. ${ }^{29}$ Numerous human studies have demonstrated safe results of administration of rhACE2 in healthy subjects and patients with ARDS. 27,30,31 Also, investigations performed in ACE2-deficient mice with severe acute lung injury have elaborated positive results of rhACE2 administration in mitigating the pulmonary problems resulting from acute lung injury (ALI). ${ }^{32}$ Furthermore, severe studies indicated the elevated levels of IL-6 in COVID-19 patients, which is related to the progression of the infection, ${ }^{33,34}$ thereby establishing the therapeutic potential of rhACE2, considering its role in the regulation of inflammatory cytokines. ${ }^{31}$ 


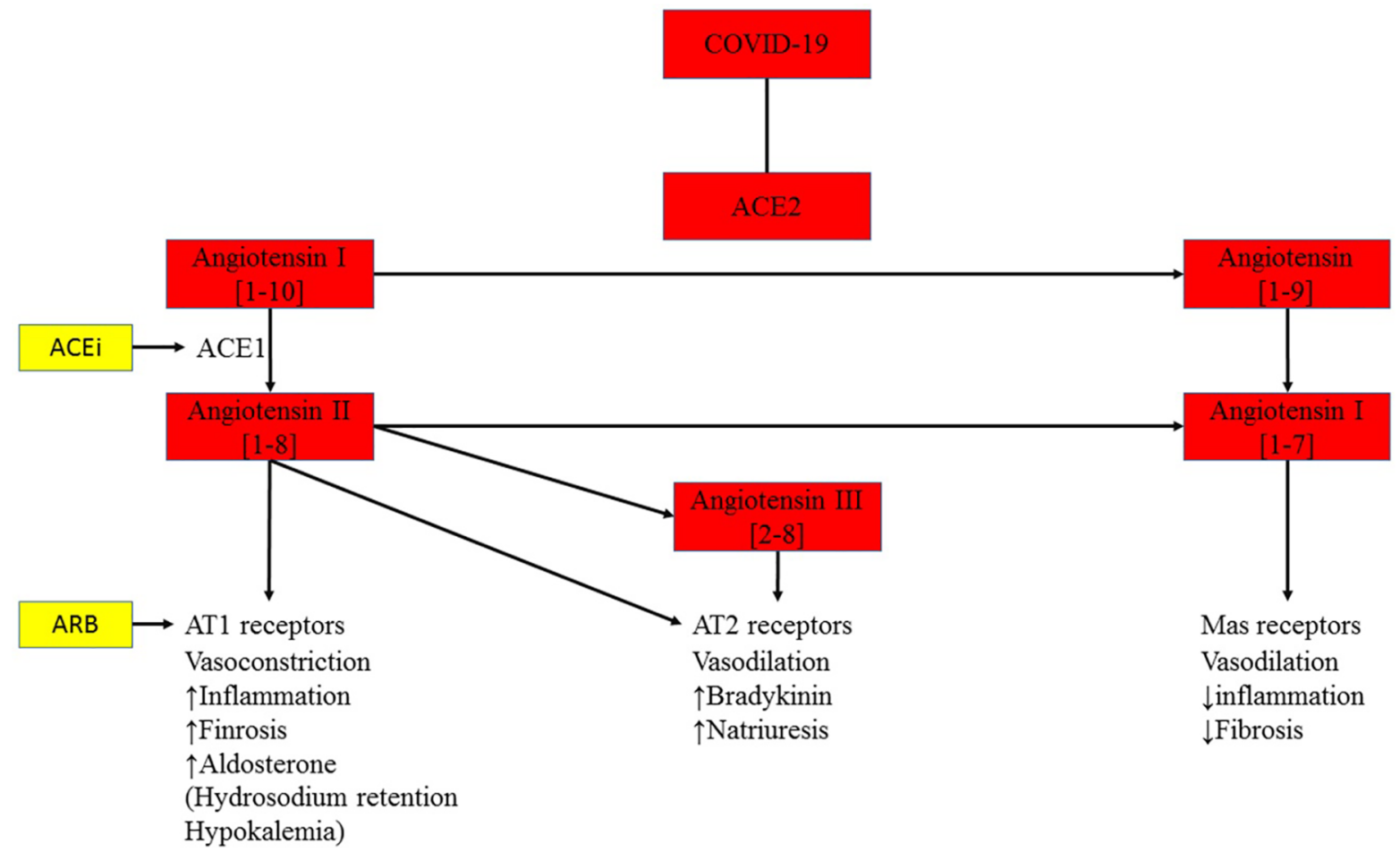

Fig. 1 The renin-angiotensin-aldosterone system in context to coronavirus disease 2019 (COVID-19) infection. The ACE2-mediated conversion of angiotensin II to angiotensin-(1-7), which opposes the actions mediated by angiotensin II via Mas receptors as well as action of ACE inhibitors (ACEi), in blocking ACE and angiotensin II receptor blockers (ARBs) to inhibit the actions of angiotensin II.

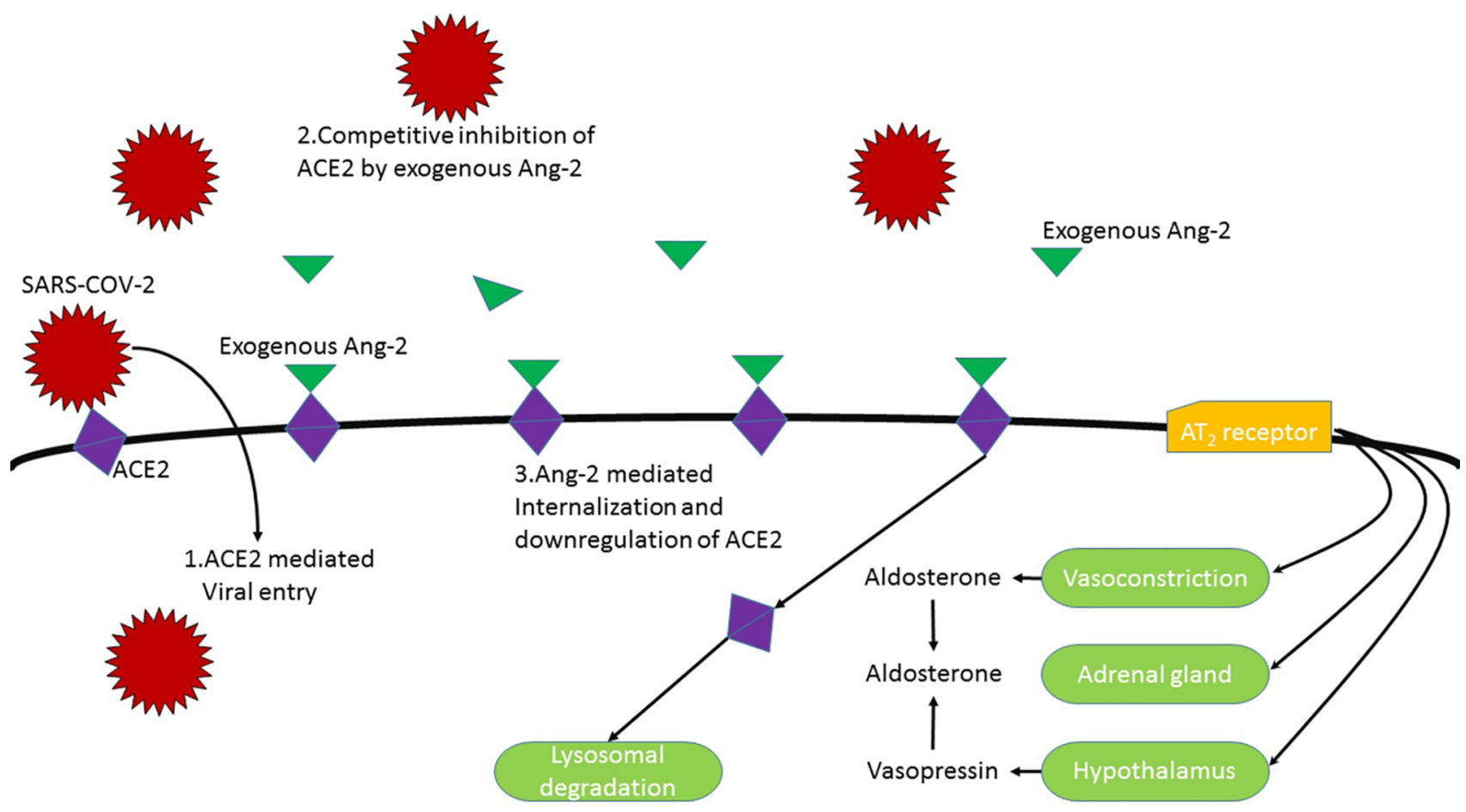

Fig. 2 Effects mediated by angiotensin II on binding of severe acute respiratory syndrome coronavirus 2 (SARS-CoV-2) and renin-angiotensin-aldosterone system (RAAS). Angiotensin binding to AT1 receptors mediated the release of aldosterone from adrenal glands and vasopressin from hypothalamus, resulting in vasoconstriction. Angiotensin II is metabolized to angiotensin-(1-7) by ACE2, which binds to SARS-CoV-2 and allows its entry into the host cell. Exogenous angiotensin II may also bind to ACE2, resulting in competitive inhibition ofACE2 receptor. Furthermore, angiotensin II mediates internalization, downregulation, and degradation of ACE2, thus preventing entry of the virus. 
- Functionally ACE (in context to ACE2; it can also be called ACE1 for convenience of understanding) generates the following:

- Angiotensin I: Asp ${ }^{1}-\mathrm{Arg}^{2}-\mathrm{Val}^{3}-\mathrm{Tyr}^{4}{ }^{4} \mathrm{Ile}^{5}-\mathrm{His}^{6}-\mathrm{Pro}^{7}-\mathrm{Phe}^{8}-\mathrm{His}^{9}{ }^{9} \mathrm{Leu}^{10}$.

- Angiotensin II: Asp1-Arg' $-\mathrm{Val}^{3}-\mathrm{Tyr}^{4}-\mathrm{Ile}^{5}-\mathrm{His}^{6}-\mathrm{Pro}^{7}-\mathrm{Phe}^{8}$ (1-8).

- Angiotensin III: $\mathrm{Arg}^{2}-\mathrm{Val}^{3}-\mathrm{Tyr}^{4}-\mathrm{Ile}^{5}-\mathrm{His}^{6}-\mathrm{Pro}^{7}-\mathrm{Phe}^{8}(7$ amino acids).

- When ACE2 acts on Ang I, it converts it into Ang 1-9.

Although ACE generates Ang II (1-8) from Ang I (1-10), ACE2 degrades Ang II (1-8) to generate the heptapeptide Ang 1-7. Ang 1-7 acts as a ligand for Mas receptor which is a GPCR.

\section{Protective Role of ACE2 in Women and Children}

ACE2 is considered as a keyhole guarding the entry of SARS-CoV-2 into the host cell. Yet, paradoxically certain studies have supported ACE2 to be an ally to fight against the COVID-19 infection, instead of being an obvious molecular target to facilitate SARS-CoV-2 entry in tissues. This has been observed by retardation of lung injury induced by treatment with recombinant ACE2 administration. This leaves us with an important question of what exactly is the role of ACE2 in COVID-19 infection. This paradox can be explained on account of posttranslational events which regulate the level of proteins in the body and maintenance of balance between the soluble and membrane-bound concentrations of ACE2. ${ }^{8}$ ACE2 can exhibit "shedding" from endothelial cells mediated by ADAM17 (a disintegrin and metalloproteinase 17), which leads to the release of the ectodomain with a bioactive and catalytic power into the circulation. ${ }^{35}$ Researchers in 2014 found the protective effects of circulating soluble ACE2 enzyme against lung injury induced by viruses like influenza A (H7N9). Whereas blocking the ACE2 gene in H5N1-infected mice resulted in severe lung damage, which was treated by administering human ACE2 in the infected mice. ${ }^{36}$ Furthermore, numerous clinical and preclinical studies have elaborated hemodynamic benefits of rhACE2 in pulmonary arterial hypertension. ${ }^{37}$ Some studies indicated that genetic variations in the ACE2 gene might possess the ability to affect the concentration of ACE2 in the human body. The ACE, ACE2, and neutral endopeptidase activities were measured in the plasma of 534 subjects in the Leeds Family Study. It was reported that $\sim 67 \%$ of phenotypic variations in circulating soluble ACE2 could be accounted for by genetic factors. ${ }^{38}$ The circulating soluble ACE2 tends to be greater in CC and CT genotype as compared with those in the TT genotype. It was hypothesized that a microRNA-mediated mechanism can regulate the functions of endothelial cells via posttranscriptional degradation. Furthermore, differences in the distribution and frequency of the ACE2 variants have been elaborated based on variations in races and ethnicity. ${ }^{8}$ Asian males were reported to possess greater ACE2 tissue expression as observed in a single cell RNA sequencing analysis. ${ }^{39}$ Similarly, a negative relationship was established between serum ACE2 activity and pulse pressure, body mass index (BMI), and estrogen levels in female patients with essential hypertension as per a case-control study conducted in the Chinese population. ${ }^{40}$ These observations indicated a dual protective response of circulating soluble ACE2 in potentiating cardiovascular effect and estrogen in upregulating ACE2 expression and tissue activity. ${ }^{9}$ Thus, it can be assumed that females may exhibit better protection profiles against COVID-19 in comparison to males.

Such an assumption may be true for children also, who have been shown to possess greater levels of ACE2 as compared with adults. ${ }^{41}$ The children with COVID-19 infection usually develop mild symptoms as compared with adults. In China, the COVID-19-positive children have been reported to exhibit less intensity of infection as compared with adults. ${ }^{41}$ About $0.9 \%$ of COVID-19 cases were younger than 9 years, and only $1.2 \%$ between 10 and 19 years of age, as per a study conducted in China on 1,099 patients. Similarly, Baric et al conducted a study on a mouse model in North Carolina and observed that younger animals exhibited greater resistance to COVID-19 infection, unlike older animals which demonstrated rapid progression and severity of the illness. ${ }^{42}$ Therefore, the relationship of COVID-19 disease and age of an individual is not only dependent upon the decline in the immune functioning of the body (i.e., immune senescence) but is also dependent on the plasma profile of ACE. ${ }^{8}$ Also, in the second to third trimester, elevated levels of ACE2 were observed in urine and plasma of women, along with increase in local placental/uterine production and action of ACE2, demonstrating a systemic hemodynamic role in the elevation of placental fetal blood flow and rapid growth of the fetus. ${ }^{43}$ ACE2 tends to pass through the placental barrier, allowing the mother to transfer her immunity to the fetus and other types of protective factors. ${ }^{8}$ Unlike the adults, there was no gender difference in COVID-19 infection in younger patients, as observed in studies conducted in China. ${ }^{44}$ This might be due to the effect of the degree of sexual maturation in children and adolescents. Besides the estradiol exerting modulating effects on ACE/ACE2 and AT1/AT2 receptors, ACE is also linked to the male reproductive system. The testes ACE comprises only the carboxy terminal domain, which is known to exhibit certain effects on a substrate, which are unknown, other than angiotensin I. ${ }^{45}$ The answer to why young animals or humans exhibit lesser risks of COVID-19 infections is not the "cross immunity" which is offered by the previous infection or the fact that children possess a powerful immune system which is not yet infected by the senescence process, but the actual reason to why children exhibit better resistance to SARS-CoV-2 infection as compared with adults is the unique plasma profile of ACE2 enzyme. The circulating soluble ACE2 aids the asymptomatic people and children to counteract the effects of the virus and limit its attachment to the cell target. This in turn helps in containing the infection and preventing the progression of the COVID-19, therefore serving as a possible therapeutic option in the near future. ${ }^{8}$

Also, this understanding of the relationship of ACE2 and COVID-19 could help to understand why some patients develop greater risks for SARS-CoV-2 infection, whereas others establish better resistance to the spread of the 
virus. ${ }^{46}$ Moreover, understanding the physiology of the individuals who are least affected by the virus can help in the development of a suitable treatment and vaccine strategies to contain the COVID-19 pandemic. In the previous years, ACE2 has been used as a biomarker for identifying variations in the BP of the patients, resulting in effective prediction and prevention of the occurrence of cardiac functioning. Similarly, the concentration of circulating soluble ACE2 can be considered to exert a prognostic effect aiding in monitoring COVID-19 infection. Also, understanding and analyzing the genetic polymorphism of ACE2 might play an important role in the prevention, diagnosis, and treatment of an individual. $^{8}$ Therefore, an enzyme linked immunosorbent assay (ELISA)-based accurate quantification of human soluble ACE2 should be developed to facilitate rapid screening, not only in serum and EDTA plasma but also in other fluids in the human body, like urine, tears, milk, and serum and saliva secretions. In case the hypothesis mentioned earlier is correct, ELISA and other methods of determining ACE2 may establish an economical and time-saving method to monitor COVID-19 infection at both the preclinical and clinical stages. On account of the advancements in the diagnostic approaches, during the current COVID-19 pandemic, with the progress in diagnostic agents like IgM or IgG detection, drug repurposing, immunotherapeutic techniques, and vaccine production, it would be appropriate to consider the relationship between ACE2 and progression of COVID-19 infection, which might provide an opportunity to the researchers to investigate a suitable treatment option. ${ }^{8}$

\section{Flip Side of ACE2: Paradoxical Role of Angiotensin II in COVID-19}

From the previous discussion, it is safe to assume that the RAAS may be associated with COVID-19 disease in both progression as well as the control. The general pathway of the RAAS coalition demonstrated conversion of Ang I to Ang II, under the influence of a pulmonary capillary endothelial enzyme, ACE2, whose activity is retarded in lung injury. About $40 \%$ of the critically ill COVID-19 patients had ARDS, as per the reports from China. ${ }^{42}$ Therefore, limited ACE activity is associated with increased mortality risks. Similar to SARS-CoV-1, which led to the coronavirus outbreak in 2003, the virus associated with the 2019 coronavirus pandemic (SARS-CoV-2), enters the target cells by utilizing cellular protease TMPRSS2 and ACE2. ${ }^{47}$ The glycoprotein spikes on the surface of the virus have been shown to interact with the ACE2 receptors, that too with 10 to 20 times greater affinity than the SARS-CoV- $1 .{ }^{48}$ The greater the affinity of SARS-CoV-2 for ACE2 receptor, the more the ease of transmission of the virus and greater is the spread of the pandemic. ${ }^{14}$ Numerous preclinical investigations of the nCOVs indicate a significant relationship between the degree of ACE2 expression and the extent of infection. ${ }^{49}$ Therefore, therapeutic approaches focusing on mitigating ACE2 expression may alleviate the infection caused by SARS-CoV-2. Ang II is hypothesized to act via numerous ways and inhibit the infection progression in COVID-19, by downregulation, competitive inhibition, internalization, and degradation of ACE2, resulting in abbreviation in the extent of viral infection by interfering with the entry mechanism of the virus into the host cell. ${ }^{3}$ One of the pathways of action of Ang II involves its binding to the ACE2 enzyme, leading to its degradation and conversion into angiotensin-(1-7), ${ }^{50}$ which may compete with the virus for attachment to ACE2 enzyme. Furthermore, Ang II is also reported to produce AT1R-mediated internalization and downregulation of ACE2 expression in both in vitro and in vivo models, via p38-MAPK and ERK1/2 pathways. ${ }^{51,52}$ Various investigations have demonstrated the hypothetical role of ARBs and ACE inhibitors in elevating ACE2 expression in the body, thereby enhancing the risks of SARS-CoV infection..$^{53}$ Patients with conditions like diabetes mellitus and increased BP have been reported to manifest severe form of COVID-19 infection in certain studies. ${ }^{42}$ However, this association between ACE inhibitors, ARBs, and the extent of COVID-19 infection requires further investigation. Many studies have demonstrated the relationship between SARS-CoV-2 and ACE2 receptors, indicating that elevated levels of ACE2 may increase the extent of infection and vice versa, ${ }^{44,54}$ as SARS-CoV-2 may be utilizing ACE2 of ACE2 to enter the host cells ${ }^{47}$ via enhanced ACE2 expression as a result of administration of ARBs and ACE inhibitors. ${ }^{55}$ Based on this corollary, it was thought logical to bring an attenuation of ACE2 expression by exogenous Ang $\mathrm{II}^{51,52}$ as well as high risks of COVID-19 infection in patients with hypertension. ${ }^{42}$ Ang II has also been hypothesized to provide protection in $\mathrm{nCoV}$ infection as being a major substrate of ACE2, it might compete with SARS-CoV-2 for binding onto the ACE2 enzyme (-Fig. 2). ${ }^{14}$

\section{RAAS Inhibitors and COVID-19}

ACE2 is insensitive to ACE inhibitors (ACEi), but ACE is significantly blocked by these inhibitors, resulting in inhibition of release of angiotensin II. ${ }^{53}$ Similar to ARBs, ACEi is significantly used for the treatment of hypertension in cardiovascular disorders. ${ }^{14}$ ACEi and ARBs were reported to elevate ACE2 membrane expression in vitro models. It should also be considered that the circulating soluble ACE2 levels may not be in line with their tissue activity and membrane expression as the latter concentration varies depending upon the tissue. ${ }^{14}$ Certain studies indicated limited concentrations of circulating ACE2 despite elevated levels of ACE2 membrane expression and tissue activity. ${ }^{56}$ Some investigations reported the occurrence of a compensatory response by elevated expressions of Ang II by increasing the membrane expression of ACE2. ${ }^{57}$ Thus, RAAS inhibitors may act at different levels of the system and produce variable effects on the enzymes and peptides involved in this pathway. ${ }^{14}$ The mineralocorticoid receptor antagonists and ARBs have been shown to enhance the ACE2 membrane expressions and their activity in clinical models. ${ }^{58,59}$ However, the actions of ACEi still need further investigations, on account of inconsistent results observed in animal models. ${ }^{60}$ Certain studies conducted in humans indicate that only ARBs can elevate the levels of ACE2, as ACEi block the release of Ang II. Yet, no significant conclusions have been drawn to date. ${ }^{60}$ Furthermore, ARBs enhance the potential benefits of activation of AT2R, 
by conversion of Ang II to Ang III as a result of blocking of AT1R. ${ }^{14}$ The cardiovascular diseases including heart failure, ischemic heart disease, arterial hypertension, and diabetes mellitus, were associated with enhanced membrane expression and tissue activity of ACE2, in response to increased Ang II, in animal models independent of ACEi/ARBs administration, and without COVID-19 infection. ${ }^{61,62}$ The impact of ACEi and ARBs in individuals infected with COVID-19 remains complex and unknown. ${ }^{63}$ Certain in vitro investigations have elaborated the protective role ARBs and not ACEi against COVID-19 infection. Many medical organizations like the European Society of Cardiology and French Society of Arterial Hypertension, ${ }^{64}$ along with numerous publications, ${ }^{65,66}$ have suggested the continuation of maintenance therapy to prevent mortality due to cardiovascular events in serious patients suffering from COVID-19. In a recent clinical study, it has been reported that there is no significant impact of the use of ACEi/ARBs and the risk of infection of COVID-19 or hospitalization. ${ }^{67}$ It should be considered that the impact of ARBs and ACEi in the current pandemic of COVID-19 is unknown and that this role may vary according to their mechanism of action. Various trials are being performed currently to investigate their effects in the current pandemic. Furthermore, ACE2 tissue expression in vitro is also reported to be affected by antihypertensive and cardiovascular drugs, like thiazide diuretics, dihydropyridines, mineralocorticoid receptor antagonists, and so forth. A single in vitro study demonstrated no significant impact of atenolol, a $\beta$-blocker, on ACE2 aortic tissue expression. ${ }^{68}$ No studies are found regarding the effect of loop diuretics on ACE2 tissue expression. ${ }^{14}$

\section{Role of Vitamin D on COVID-19 Infection}

Vitamin D is a lipid-soluble micronutrient that is available through food sources or synthesized by skin-mediated conversion of 7-dehydrocholesterol on exposure to ultraviolet $B$ (UVB) radiation. ${ }^{6970}$ It produces multiple cellular responses such as maintenance of phosphorous and calcium homeostasis, and the susceptibility to infectious diseases have been reported to increase in case of its deficiency. ${ }^{69-71}$ Calcitriol (1,25-dihydroxycholecalciferol) is an active form of vitamin $\mathrm{D}$ and has been attributed to play a role as an immune system regulating steroid hormone, which downregulates the expression of inflammatory cytokines and increases the activity of macrophages. ${ }^{72}$ Besides, it has been shown to also mediate the expression of antimicrobial peptides (AMP), which are located on monocytes, natural killer cells, epithelial cell lining of the respiratory tract, and neutrophils. ${ }^{73}$ It further blocks the production of T-helper 17 cells (proinflammatory) and induces the production of suppressive regulatory $\mathrm{T}$ cells. ${ }^{74}$ Furthermore, the proinflammatory peptides associated with the adaptive immune system, especially the ones involved in acute inflammatory cytokine storm, are regulated by vitamin D. . $^{70,73,75}$ Numerous epidemiological studies have demonstrated the potential link between deficiency of vitamin $\mathrm{D}$ in the body and enhanced risks of respiratory tract infections. ${ }^{76,77}$ Also, many studies reveal the protective role of vitamin D supplementation against virus mediated infections. Various actions of vitamin D contribute to its immunoregulatory effects in respiratory tract infections, like immune cell recruitment to the site of infection, disruption of the lipid envelope of the virus by LL-35, mitigating viral replication cycle, elevating the levels of virus specific CD8+ $\mathrm{T}$ cells, and increasing CXCL10 and IFN-p in airway epithelium. ${ }^{77}$ Cell culture studies support the role of vitamin D in alleviating viral infections, especially those caused by enveloped viruses. ${ }^{76}$ Therefore, the role of vitamin $\mathrm{D}$ in mitigating the progression of SARS-CoV-2, is an important area of concern, to the researchers to investigate the therapeutic potential of vitamin D in the current COVID-19 pandemic. Numerous studies reveal low levels of vitamin D in people with cardiovascular disease (CVDs), diabetes, and hypertension as well as in the geriatric population, all considered as severe comorbidities of COVID-19. ${ }^{75,78-82}$

COVID-19 is associated with the manifestation of acute lung injury (ALI) and ARDS, which are the primary cause of lung dysfunction and respiratory failure in SARS-CoV-2infected patients. ${ }^{83}$ As elaborated earlier, decrease in ACE2/Ang-(1-7) expression and elevation in the levels of ACE/Ang II, contributes to ALI. ${ }^{26}$ Thus, enhancement in the expression of vitamin D receptor and ACE2 levels may play a defensive role against ALI conditions. ${ }^{84}$ An individual participant data (IPD) meta-analysis of randomized-controlled trials revealed that supplementation of vitamin D can mitigate the risks of developing respiratory tract infections. ${ }^{70}$ Although the exact molecular mechanism of vitamin D-mediated downregulation of intrarenal RAAS is not completely understood, yet various studies have reported that calcitriol, the active metabolite of vitamin $\mathrm{D}$, can block the production of cyclic AMP response element-binding protein (CREB) and the related partner complex. ${ }^{75}$ Furthermore, the transcriptional regulatory complex is another significant downregulator of intrarenal RAAS and comprises CREB1, nuclear receptor corepressor 1 (NCOR1), and vitamin D receptor, which in turn interacts with the CRE-like domain. Thus vitamin D is considered to suppress the activity and functioning of RAAS by blocking renin and ACE/Ang II/AT1R cascade ${ }^{85}$ Deficiency in the levels of vitamin $D$ has been shown to be significantly associated with risks to develop CVDs along with obesity, diabetes, and hypertriglyceridemia, as per the analysis report of the third National Health and Nutrition Examination Survey (NHANES 3 1988-1994). ${ }^{11}$ Vitamin D is reported to upregulate the ACE2 expression and downregulate the renin ACE levels, as observed in animal studies. ${ }^{86}$ The results obtained in the case of vitamin D deficiency were opposite to the results mentioned earlier. ${ }^{75} \mathrm{~A}$ relationship has been observed between vitamin D deficiency and elevation in the actions of RAAS and renin, and enhanced levels of Ang II, in numerous human cross-sectional studies. ${ }^{87}$ Oral supplementation of vitamin D has been found to reduce diastolic BP in hypertensive patients. ${ }^{75}$ Moreover, an investigational study performed on 494 women demonstrated that vitamin D deficiency along with elevated PTH levels were independent predictors of insulin resistance, hyperglycemia, and dysfunction of pancreatic $\beta$-cells. ${ }^{88}$ Vitamin D deficiency is considered to induce such effects to elevate $G$ protein RhoA and ROS 
production by enhancing the synthesis of Ang II and renin, resulting in blockage of pathways required for intracellular glucose transporter, further developing metabolic disorder and insulin resistance. ${ }^{87}$ Vitamin $\mathrm{D}$ regulates the secretion of insulin via calcium-level regulation within the cell ${ }^{89}$ and is also associated with the production and secretion of insulin in the pancreas via the PTH link.

In the clinical and in vitro studies vitamin $\mathrm{D}$ has been found to have a protective influence against both the respiratory (influenza, coronavirus, pneumonia) ${ }^{90}$ and nonrespiratory viruses (human immunodeficiency virus [HIV], hepatitis C virus [HCV], dengue virus). ${ }^{91}$ Therefore, the ability of vitamin $D$ to upregulate AMPs and mediate interference of antiviral cytokines with the viral replication cycle establishes its role in viral infections. ${ }^{92}$ Vitamin $\mathrm{D}$ is responsible for downregulating the expression of host cell receptors, required for binding of the virus to enter the host cell. ${ }^{10}$ Vitamin D functions as an effective negative RAAS regulator, via canonical vitamin $D$ receptor pathway, resulting in RAAS suppression and upregulation of ACE2 expression both in vitro and in vivo. ${ }^{93}$ Various studies reveal that besides downregulating the receptors for viral attachment, vitamin D is also responsible for fine tuning of the viral induced proinflammatory responses. ${ }^{94}$ The pulmonary damage in a model of ALI and ARDS, induced by deficiency in vitamin $\mathrm{D}$, is associated with RAAS and ACE2 receptor modulation. ${ }^{85,95}$ Also, the subtropical areas with limited sunlight exposure and vitamin D deficiency (Northern US and Europe), have been associated with greater risks of respiratory viruses, like influenza and corona viruses. ${ }^{96}$ Hence, given the ongoing COVID-19 pandemic, summarizing all the aspects related to vitamin D which may act as a shield against the entry of the virus by maintaining tight gap junctions, increasing innate immunity via recruitment of anti-viral and anti-microbial peptides, modulating $\mathrm{T}$ cell response through adaptive immunity, therefore, playing a pivotal role in the anti-inflammatory response, and many more as described above, all of which contribute to its effect in alleviating disease severity in SARS-CoV-2 infected patients.

\section{Conclusion}

The COVID-19 pandemic has created havoc across the world, posing immense threats and challenges to the healthcare professionals compelling to them, to develop appropriate vaccines and treatment strategies to control the infection caused by SARS-CoV-2. The review elaborates the opportunity to utilize the hypothesis regarding the role of ACE2/vitamin D in COVID-19 infection in mitigating the progression of the disease. The active form of vitamin $D$ (calcitriol) is shown to inhibit the production of CREB protein and the related partner complex. Moreover, the efficiency of vitamin D supplementation in high risk groups of COVID-19 is also elaborated in the review. Vitamin D supplementation provides an immediate, natural, economical, and accessible strategy to fight the current pandemic. The conventional oral supplementation of vitamin $\mathrm{D}$ is considered to significantly aim at restricting COVID-19 infection by upregulating ACE2 expression, thus alleviating the cytokine storm responsible for the severity of the disease. As mounting evidence supports the increased expression of ACE2 mRNA by vitamin D action, the possibility of ACE 2 being a downstream target of vitamin D may be further explored. Although no significant studies have elaborated the therapeutic potential of vitamin D so far, yet the review extends awareness and opportunity for the researchers to develop vitamin D supplementation into a new "at hand tool," to minimize and control the accelerated spread of the infection across the globe. Therefore, a clear understanding of the activity pattern of ACE2 and the associated role of vitamin $\mathrm{D}$ in mitigating the SARS-CoV-2 infection provides a fundamental opportunity for researchers all over the globe to explore this hypothesis, and develop an effective treatment strategy to aid in the global fight against COVID-19 pandemic.

\section{Conflict of Interest}

None declared.

\section{References}

1 Mackenzie JS, Smith DW. COVID-19: a novel zoonotic disease caused by a coronavirus from China: what we know and what we don't. Microbiol Aust 2020;MA20013:MA20013

2 World Health Organization. Statement on the meeting of the International Health Regulations (2005) Emergency Committee regarding the outbreak of novel coronavirus (2019-nCoV). Available at: https://www.worldometers. info/coronavirus/?utm_campaign=homeAdUOA?Si. Published January 23, 2020

3 Busse LW, Chow JH, McCurdy MT, Khanna AK. COVID-19 and the RAAS-a potential role for angiotensin II? Crit Care 2020;24(1):136

4 Hall A, Busse LW, Ostermann M. Angiotensin in critical care. Crit Care 2018;22(1):69

5 Singh AK, Gupta R, Misra A. Comorbidities in COVID-19: outcomes in hypertensive cohort and controversies with renin angiotensin system blockers. Diabetes Metab Syndr 2020;14(4):283-287

6 Wang D, Hu B, Hu C, et al. Clinical characteristics of 138 hospitalized patients with 2019 novel coronavirus-infected pneumonia in Wuhan, China. JAMA 2020;323(11):1061-1069

7 Murthy S, Gomersall CD, Fowler RA. Care for critically ill patients with COVID-19. JAMA 2020;323(15):1499-1500

8 Ciaglia E, Vecchione C, Puca AA. COVID-19 infection and circulating ACE2 levels: protective role in women and children. Front Pediatr 2020;8:206

9 da Silva JS, Gabriel-Costa D, Wang H, et al. Blunting of cardioprotective actions of estrogen in female rodent heart linked to altered expression of cardiac tissue chymase and ACE2. J Renin Angiotensin Aldosterone Syst 2017;18(3):1470320317722270

10 Arboleda J, Silvio U. Vitamin D supplementation: a potential approach for COVID-19 therapeutics? Front Immunol 2020;11 (4):1523

11 Honardoost M, Ghavideldarestani M, Khamseh ME. Role of vitamin $\mathrm{D}$ in pathogenesis and severity of COVID-19 infection. Arch Physiol Biochem 2020;1-7

12 Donoghue $\mathrm{M}$, Hsieh $\mathrm{F}$, Baronas $\mathrm{E}$, et al. A novel angiotensin-converting enzyme-related carboxypeptidase (ACE2) converts angiotensin I to angiotensin 1-9. Circ Res 2000;87(5):E1-E9

13 Tipnis SR, Hooper NM, Hyde R, Karran E, Christie G, Turner AJ. A human homolog of angiotensin-converting enzyme. Cloning and functional expression as a captopril-insensitive carboxypeptidase. J Biol Chem 2000;275(43):33238-33243 
14 Turner AJ, Tipnis SR, Guy JL, Rice G, Hooper NM. ACEH/ACE2 is a novel mammalian metallocarboxypeptidase and a homologue of angiotensin-converting enzyme insensitive to ACE inhibitors. Can J Physiol Pharmacol 2002;80(4):346-353

15 Vickers C, Hales P, Kaushik V, et al. Hydrolysis of biological peptides by human angiotensin-converting enzyme-related carboxypeptidase. J Biol Chem 2002;277(17):14838-14843

16 Choi HS, Kim IJ, Kim CS, et al. Angiotensin-[1-7] attenuates kidney injury in experimental Alport syndrome. Sci Rep 2020;10(1):4225

17 Karnik SS, Unal H, Kemp JR, et al. International union of basic and clinical pharmacology. XCIX. Angiotensin receptors: interpreters of pathophysiological angiotensinergic stimuli [corrected]. Pharmacol Rev 2015;67(4):754-819

18 Santos RAS, Sampaio WO, Alzamora AC, et al. The ACE2/angiotensin-(1-7)/MAS axis of the renin-angiotensin system: focus on angiotensin-(1-7) Physiol Rev 2018;98(1):505-553

19 Kawai T, Takayanagi T, Forrester SJ, et al. Vascular ADAM17 (a disintegrin and metalloproteinase domain 17) is required for angiotensin II/ $\beta$-aminopropionitrile-induced abdominal aortic aneurysm. Hypertension 2017;70(5):959-963

20 Kostenis E, Milligan G, Christopoulos A, et al. G-protein-coupled receptor Mas is a physiological antagonist of the angiotensin II type 1 receptor. Circulation 2005;111(14):1806-1813

21 Sparks MA, Crowley SD, Gurley SB, Mirotsou M, Coffman TM. Classical renin-angiotensin system in kidney physiology. Compr Physiol 2014;4(3):1201-1228

22 Hamming I, Timens W, Bulthuis ML. Lely AT, Navis G, van Goor $\mathrm{H}$. Tissue distribution of ACE2 protein, the functional receptor for SARS coronavirus. A first step in understanding SARS pathogenesis. J Pathol 2004;203(2):631-637

23 Dandona P, Dhindsa S, Ghanim H, Chaudhuri A. Angiotensin II and inflammation: the effect of angiotensin-converting enzyme inhibition and angiotensin II receptor blockade. J Hum Hypertens 2007;21(1):20-27

24 Monteil V, Kwon H, Prado $\mathrm{P}$, et al. Inhibition of SARS-CoV-2 infections in engineered human tissues using clinical-grade soluble human ACE2. Cell 2020;181(4):905913.e7

25 Huang C, Wang Y, Li X, et al. Clinical features of patients infected with 2019 novel coronavirus in Wuhan, China. Lancet 2020;395(10223):497-506

26 Kuba K, Imai Y, Rao S, et al. A crucial role of angiotensin converting enzyme 2 (ACE2) in SARS coronavirus-induced lung injury. Nat Med 2005;11(8):875-879

27 Zhang H, Baker A. Recombinant human ACE2: acing out angiotensin II in ARDS therapy. Crit Care 2017;21(1):305

28 Guo J, Huang Z, Lin L, Lv J. Coronavirus disease 2019 (covid-19) and cardiovascular disease: a viewpoint on the potential influence of angiotensin-converting enzyme inhibitors/angiotensin receptor blockers on onset and severity of severe acute respiratory syndrome coronavirus 2 infection. J Am Heart Assoc 2020;9(7):e016219

29 Colafella KMM, Uijl E, Danser J, Interference with the renin-angiotensin system (RAS): classical inhibitors and novel approaches. Biomedical Sciences: Encyclopedia of Endocrine Diseases 2018. Doi: 10.1016/B978-0-12-801238-3.65341-2

30 Haschke M, Schuster M, Poglitsch M, et al. Pharmacokinetics and pharmacodynamics of recombinant human angiotensin-converting enzyme 2 in healthy human subjects. Clin Pharmacokinet 2013;52(9):783-792

31 Khan A, Benthin C, Zeno B, et al. A pilot clinical trial of recombinant human angiotensin-converting enzyme 2 in acute respiratory distress syndrome. Crit Care 2017;21(1):234

32 Sriram K, Insel PA. A hypothesis for pathobiology and treatment of COVID-19: The centrality of ACE1/ACE2 imbalance. Br J Pharmacol 2020;177(21):4825-4844
33 Wu C, Chen X, Cai Y, et al. Risk factors associated with acute respiratory distress syndrome and death in patients with coronavirus disease 2019 pneumonia in Wuhan, China. JAMA Intern Med 2020;180(7):934-943

34 prpt. Wan S, Yi Q Fan S, et al. Characteristics of lymphocyte subsets and cytokines in peripheral blood of 123 hospitalized patients with 2019 novel coronavirus pneumonia (NCP). MedRxiv 2020. Doi: 10.1101/2020.02.10.20021832./prpt

35 Heurich A, Hofmann-Winkler H, Gierer S, Liepold T, Jahn O, Pöhlmann S. TMPRSS2 and ADAM17 cleave ACE2 differentially and only proteolysis by TMPRSS2 augments entry driven by the severe acute respiratory syndrome coronavirus spike protein. J Virol 2014;88(2):1293-1307

36 Yang $\mathrm{P}, \mathrm{Gu} \mathrm{H}, \mathrm{Zhao} \mathrm{Z}$, et al. Angiotensin-converting enzyme 2 (ACE2) mediates influenza H7N9 virus-induced acute lung injury. Sci Rep 2014;4:7027

37 Hemnes AR, Rathinasabapathy A, Austin EA, et al. A potential therapeutic role for angiotensin-converting enzyme 2 in human pulmonary arterial hypertension. Eur Respir J 2018;51(6):1702638

38 Rice GI, Jones AL, Grant PJ, Carter AM, Turner AJ, Hooper NM. Circulating activities of angiotensin-converting enzyme, its homolog, angiotensin-converting enzyme 2, and neprilysin in a family study. Hypertension 2006;48(5):914-920

39 prpt. Zhao Y, Zhao Z, Wang Y, Zhou Y, Ma Y, Zuo W. Single-cell RNA expression profiling of ACE2, the putative receptor of Wuhan 2019-nCoV. bioRxiv 2020;919985./prpt

40 Zhang Q, Cong $\mathrm{M}$, Wang $\mathrm{N}$, et al. Association of angiotensin-converting enzyme 2 gene polymorphism and enzymatic activity with essential hypertension in different gender: a case-control study. Medicine (Baltimore) 2018;97(42):e12917

41 Bénéteau-Burnat B, Baudin B, Morgant G, Baumann FC, Giboudeau J. Serum angiotensin-converting enzyme in healthy and sarcoidotic children: comparison with the reference interval for adults. Clin Chem 1990;36(2):344-346

42 Guan WJ, Ni ZY, Hu Y, et al; China Medical Treatment Expert Group for Covid-19. Clinical characteristics of coronavirus disease 2019 in China. N Engl J Med 2020;382(18):1708-1720

43 Ghadhanfar E, Alsalem A, Al-Kandari S, Naser J, Babiker $\mathrm{F}$, Al-Bader M. The role of ACE2, angiotensin-(1-7) and Mas1 receptor axis in glucocorticoid-induced intrauterine growth restriction. Reprod Biol Endocrinol 2017;15(1):97

44 Dong Y, Mo X, Hu Y, et al. Epidemiological characteristics of 2143 pediatric patients with 2019 coronavirus disease in China. Pediatrics 2020. Doi 10.1542/peds.2020-0702

45 Li LJ, Zhang FB, Liu SY, et al. Human sperm devoid of germinal angiotensin-converting enzyme is responsible for total fertilization failure and lower fertilization rates by conventional in vitro fertilization. Biol Reprod 2014;90(6):125

46 Ingraham NE, Barakat AG, Reilkoff R, et al. Understanding the renin-angiotensin-aldosterone-SARS-CoV axis: a comprehensive review. Eur Respir J 2020;56(1):2000912

47 Hoffmann M,Kleine-WeberH,SchroederS, etal.SARS-CoV-2 cell entry depends on ACE2 and TMPRSS2 and is blocked by a clinically proven protease inhibitor. Cell 2020;181(2):271-280.e8

48 Wrapp D, Wang N, Corbett KS, et al. Cryo-EM structure of the 2019-nCoV spike in the prefusion conformation. Science 2020;367(6483):1260-1263

49 Hofmann H, Geier M, Marzi A, et al. Susceptibility to SARS coronavirus $\mathrm{S}$ protein-driven infection correlates with expression of angiotensin converting enzyme 2 and infection can be blocked by soluble receptor. Biochem Biophys Res Commun 2004;319(4):1216-1221

50 Chawla LS, Chen S, Bellomo R, Tidmarsh GF. Angiotensin converting enzyme defects in shock: implications for future therapy. Crit Care 2018;22(1):274 
51 Koka V, Huang XR, Chung AC, Wang W, Truong LD, Lan HY. Angiotensin II up-regulates angiotensin I-converting enzyme (ACE), but down-regulates ACE2 via the AT1-ERK/p38 MAP kinase pathway. Am J Pathol 2008;172(5):1174-1183

52 Fernandes T, Hashimoto NY, Magalhães FC, et al. Aerobic exercise training-induced left ventricular hypertrophy involves regulatory MicroRNAs, decreased angiotensin-converting enzyme-angiotensin II, and synergistic regulation of angiotensin-converting enzyme 2-angiotensin (1-7) Hypertension 2011;58(2):182-189

53 Ferrario CM, Jessup J, Chappell MC, et al. Effect of angiotensin-converting enzyme inhibition and angiotensin II receptor blockers on cardiac angiotensin-converting enzyme 2 . Circulation 2005;111(20):2605-2610

54 Li W, Moore MJ, Vasilieva N, et al. Angiotensin-converting enzyme 2 is a functional receptor for the SARS coronavirus. Nature 2003;426(6965) :450-454

55 Ishiyama Y, Gallagher PE, Averill DB, Tallant EA, Brosnihan KB, Ferrario $\mathrm{CM}$. Upregulation of angiotensin-converting enzyme 2 after myocardial infarction by blockade of angiotensin II receptors. Hypertension 2004;43(5):970-976

56 Danser AHJ, Epstein M, Batlle D. Renin-angiotensin system blockers and the COVID-19 pandemic: at present there is no evidence to abandon renin-angiotensin system blockers. Hypertension 2020;75(6):1382-1385

57 Mourad JJ, Levy BI. Interaction between RAAS inhibitors and ACE2 in the context of COVID-19. Nat Rev Cardiol 2020;17(5):313

58 Keidar S, Gamliel-Lazarovich A, Kaplan M, et al. Mineralocorticoid receptor blocker increases angiotensinconverting enzyme 2 activity in congestive heart failure patients. Circ Res 2005;97(9):946-953

59 Zhong JC, Ye JY, Jin HY, et al. Telmisartan attenuates aortic hypertrophy in hypertensive rats by the modulation of ACE2 and profilin-1 expression. Regul Pept 2011;166(1-3):90-97

60 Sriram K, Insel PA. Risks of ACE Inhibitor and ARB Usage in COVID-19: Evaluating the Evidence. Clin Pharmacol Ther 2020;108(2):236-241

61 Goulter AB, Goddard MJ, Allen JC, Clark KL.. ACE2 gene expression is up-regulated in the human failing heart. BMC Med 2004;2-19

62 Anguiano L, Riera M, Pascual J, Soler MJ. Circulating ACE2 in cardiovascular and kidney diseases. Curr Med Chem 2017;24(30):3231-3241

63 Aronson JK, Ferner RE. Drugs and the renin-angiotensin system in covid-19. BMJ 2020;369:m1313

64 Position statement of the ESC council on hypertension on ace inhibitors and angiotensin receptor blockers. https:// www.escardio.org/Councils/Council-on-Hypertension-(CHT)/ News/positionstatement-of-the-esc-council-on-hypertension-on-aceinhibitors-and-... Published 2020. Accessed Position statement of the ESC council on hypertension on ace inhibitors and angiotensin receptor blockers. https://www.escardio. org/Councils/Council-on-Hypertension-(CHT)/News/position statement-of-the-esc-council-on-hypertension-on-aceinhibitors-and-... Published 2020. Accessed 2020

65 Rico-Mesa JS, White A, Anderson AS. Outcomes in patients with COVID-19 infection taking ACEI/ARB. Curr Cardiol Rep 2020;22(5):31

66 Kuster GM, Pfister O, Burkard T, et al. SARS-CoV2: should inhibitors of the renin-angiotensin system be withdrawn in patients with COVID-19? Eur Heart J 2020;41(19):1801-1803

67 prpt. Dublin S, Walker RL, Floyd JS, et al. Renin-angiotensin-aldosterone system inhibitors and COVID-19 infection or hospitalization: a cohort study. medRxiv. 2020:2020./prpt
68 Igase $M$, Strawn $W B$, Gallagher PE, Geary RL, Ferrario CM. Angiotensin II AT1 receptors regulate ACE2 and angiotensin-(1-7) expression in the aorta of spontaneously hypertensive rats. Am J Physiol Heart Circ Physiol 2005;289(3):H1013-H1019

69 Pilz S, Tomaschitz A, Ritz E, Pieber TR. Vitamin D status and arterial hypertension: a systematic review. Nat Rev Cardiol 2009;6(10):621-630

70 Martineau AR, Jolliffe DA, Hooper RL, et al. Vitamin D supplementation to prevent acute respiratory tract infections: systematic review and meta-analysis of individual participant data. BMJ 2017;356:i6583

71 Yamshchikov AV, Desai NS, Blumberg HM, Ziegler TR, Tangpricha V. Vitamin D for treatment and prevention of infectious diseases: a systematic review of randomized controlled trials. Endocr Pract 2009;15(5):438-449

72 Di Rosa M, Malaguarnera M, Nicoletti F, Malaguarnera L. Vitamin D3: a helpful immuno-modulator. Immunology 2011;134(2):123-139

73 Mawson AR. Role of fat-soluble vitamins A and D in the pathogenesis of Influenza: a new perspective. Int Sch Res Notices. 2013 Doi: $10.5402 / 2013 / 246737$

74 Yang J, Jun X, Zhang H. Effect of vitamin D on ACE2 and vitamin $D$ receptor expression in rats with LPS-induced acute lung injury. Zhonghua Jizhen Yixue Zazhi 2016;25:1284-1289

75 Andersen LB, Przybyl L, Haase N, et al. Vitamin D depletion aggravates hypertension and target-organ damage. J Am Heart Assoc 2015;4(2):e001417

76 Beard JA, Bearden A, Striker R. Vitamin D and the anti-viral state. J Clin Virol 2011;50(3):194-200

77 Teymoori-Rad M, Shokri F, Salimi V, Marashi SM. The interplay between vitamin D and viral infections. Rev Med Virol 2019;29(2):e2032

78 Grant WB, Lahore H, McDonnell SL, Baggerly CA, French CB, Aliano JL, Bhattoa HP. Vitamin D supplementation could prevent and treat influenza, coronavirus, and pneumonia infections. Nutrients 2020;12(5):988

79 Kheiri B, Abdalla A, Osman M, Ahmed S, Hassan M, Bachuwa G. Vitamin D deficiency and risk of cardiovascular diseases: a narrative review. Clin Hypertens 2018;24:9

80 Hughes DA, Norton R. Vitamin D and respiratory health. Clin Exp Immunol 2009;158(1):20-25

81 Berridge MJ. Vitamin D deficiency and diabetes. Biochem J 2017;474(8):1321-1332

82 Nakashima A, Yokoyama K, Yokoo T, Urashima M. Role of vitamin $\mathrm{D}$ in diabetes mellitus and chronic kidney disease. World J Diabetes 2016;7(5):89-100

83 Li X, Ma X. Acute respiratory failure in COVID-19: is it "typical" ARDS? Crit Care 2020;24(1):198

84 Ferrario CM. ACE2: more of Ang-(1-7) or less Ang II? Curr Opin Nephrol Hypertens 2011;20(1):1-6

85 Xu J, Yang J, Chen J, Luo Q Zhang Q Zhang H. Vitamin D alleviates lipopolysaccharide-induced acute lung injury via regulation of the renin-angiotensin system. Mol Med Rep 2017;16(5):7432-7438

86 Report of the WHO-China Joint Mission on Coronavirus Disease 2019 (COVID-19). Available at: https://wwwwhoint/docs/default-source/coronaviruse/who-china-joint-mission-on-covid-19-final-reportpdf. Published February 2020. Accessed April 7, 2020

87 Mozos I, Marginean O. Links between vitamin D deficiency and cardiovascular diseases. BioMed Res Int 2015;2015:109275

88 Kayaniyil S, Vieth R, Retnakaran R, et al. Association of vitamin $D$ with insulin resistance and $\beta$-cell dysfunction in subjects at risk for type 2 diabetes. Diabetes Care 2010;33(6):1379-1381 
89 Sung CC, Liao MT, Lu KC, Wu CC. Role of vitamin D in insulin resistance. J Biomed Biotechnol 2012;2012:634195

90 Jayawardena R, Sooriyaarachchi P, Chourdakis M, Jeewandara C, Ranasinghe P. Enhancing immunity in viral infections, with special emphasis on COVID-19: a review. Diabetes Metab Syndr 2020;14(4):367-382

91 Campbell GR, Spector SA. Vitamin D inhibits human immunodeficiency virus type 1 and Mycobacterium tuberculosis infection in macrophages through the induction of autophagy. PLoS Pathog 2012;8(5):e1002689

92 Schögler A, Muster RJ, Kieninger E, et al. Vitamin D represses rhinovirus replication in cystic fibrosis cells by inducing LL-37. Eur Respir J 2016;47(2):520-530
93 Ajabshir S, Asif A, Nayer A. The effects of vitamin D on the renin-angiotensin system. J Nephropathol 2014;3(2):41-43

94 Arboleda Alzate JF, Rodenhuis-Zybert IA, Hernández JC, Smit JM, Urcuqui-Inchima S. Human macrophages differentiated in the presence of vitamin D3 restrict dengue virus infection and innate responses by downregulating mannose receptor expression. PLoS Negl Trop Dis 2017;11(10):e0005904

95 Agarwal R. Are vitamin D receptor agonists like angiotensin-converting enzyme inhibitors without side effects? Kidney Int 2010;77(11):943-945

96 Davidson BL, Alansari K. Vitamin D deficiency can impair respiratory health. Respirology 2018;23(6):554-555 